\title{
ON A SUBCLASS OF SPIRAL-LIKE FUNCTIONS
}

\author{
E. M. SILVIA
}

Abstract. Let $\alpha \geqq 0,0 \leqq \beta<1,|\lambda|<\pi / 2$ and suppose that $f(z)=z+\sum_{n=2}^{\infty} a_{n} z^{n}$ is holomorphic in $U=\{z:|z|<1\}$. If

$$
\operatorname{Re}\left[e^{i \lambda} \frac{z f^{\prime}(z)}{f(z)}+\alpha\left(\frac{z f^{\prime \prime}(z)}{f^{\prime}(z)}+1-\frac{z f^{\prime}(z)}{f(z)}\right)\right]>\beta \cos \lambda
$$

for $z \in U$, then $f(z)$ is said to be $\alpha$ - $\lambda$-spiral-like of order $\beta$ and we write $f(z) \in S_{\alpha}^{\lambda}(\beta)$. The author shows that for each $\alpha \geqq 0, \alpha-\lambda$ spiral-like functions of order $\beta$ are $\lambda$-spiral-like of order $\beta$. The following representation theorem is obtained: The function $f(z) \in S_{\alpha}^{\lambda}(\beta)(\alpha>0,0 \leqq \beta<1,|\lambda|<\pi / 2)$, if and only if there exists a function $F(\zeta) \lambda$-spiral-like of order $\beta$ such that

$$
F(z)=\left[\left(e^{i \lambda / \alpha}\right) \int_{0}^{z} F(\zeta)^{i \lambda / \alpha \zeta^{-1}} d \zeta\right]^{\alpha e^{-i \lambda}} .
$$

A distortion theorem for $\log |f(z) / z|$ and a rotation theorem for $\arg f(z) / z$ are also proved for functions $f(z) \in S_{0}^{\lambda}(\beta)$.

1. Let $A$ denote the class of functions normalized by $f(z)=z+$ $\sum_{n=2}^{\infty} a_{n} z^{n}$ which are analytic in $U(|z|<1)$. For $0 \leqq \beta<1$, we will let $S^{*}(\beta)$ represent the class of functions contained in $A$ which are univalent and starlike of order $\beta$; i.e., $f(z) \in S^{*}(\beta)$ if $f(z)=z+\sum_{n=2}^{\infty} a_{n} z^{n}$ is analytic and univalent satisfying $\operatorname{Re} z f^{\prime}(z) \mid f(z)>\beta(z \in U)$. Also, let $P$ denote the class of analytic functions normalized by $p(z)=1+\sum_{n=1}^{\infty} c_{n} z^{n}$ such that $\operatorname{Re} p(z)>0(z \in U)$.

A function $f(z) \in A$ is said to be spiral-like if there exists a $\lambda(|\lambda|<\pi / 2)$ such that $\operatorname{Re} e^{i \lambda} z f^{\prime}(z) \mid f(z)>0(z \in U)$. L. Spaček defined the class of spiral-like functions in 1933 and showed that these functions are univalent [15].

In 1967, R. Libera [6] extended this definition to functions spiral-like of order $\beta$. We say that $f(z) \in A$ is $\lambda$-spiral-like of order $\beta(0 \leqq \beta<1$, $|\lambda|<\pi / 2)$ if $\operatorname{Re} e^{i \lambda} z f^{\prime}(z) / f(z)>\beta \cos \lambda(z \in U)$.

A function $f(z) \in A$ satisfying $f(z) f^{\prime}(z) \neq 0 \quad(0<|z|<1)$ is said to be $\alpha$-starlike of order $\beta(\alpha \geqq 0,0 \leqq \beta<1)$ if

$$
\operatorname{Re}\left\{(1-\alpha) \frac{z f^{\prime}(z)}{f(z)}+\alpha\left(\frac{z f^{\prime \prime}(z)}{f^{\prime}(z)}+1\right)\right\}>\beta \quad(z \in U) .
$$

Received by the editors March 1, 1973.

AMS (MOS) subject classifications (1970). Primary 30A36; Secondary 30A32.

Key words and phrases. Univalent, $\alpha$-starlike, $\lambda$-spiral-like.

(c) American Mathematical Society 1974 
For $\beta=0$, we have the class of $\alpha$-starlike functions (of order zero) which has been thoroughly investigated in [7], [8], [9], [10], and [11]. Some of these results have been extended to $0<\beta<1$ by the author [13].

In this note, a class of functions which contains the classes of $\alpha$-starlike functions of order $\beta$ and $\lambda$-spiral-like functions of order $\beta$ as special cases is defined; the functions in this new class will be shown to be $\lambda$-spiral-like. The author obtains an integral representation for the elements of this class in terms of $\lambda$-spiral-like functions of order $\beta$. Finally, a distortion and a rotation theorem for $f(z) / z$ whenever $f(z)$ is in this class is proved.

2. Just as the definition of $\lambda$-spiral-likeness of order $\beta$ generalizes the definition of starlikeness of order $\beta$, we will generalize the definition of $\alpha$-starlikeness of order $\beta$ to $\alpha-\lambda$-spiral-likeness of order $\beta$. In this section, we define the class of $\alpha$ - $\lambda$-spiral-like functions of order $\beta$-denoted $S_{\alpha}^{\lambda}(\beta)$-and show that each $f(z) \in S_{\alpha}^{\lambda}(\beta)$ is $\lambda$-spiral-like of order $\beta$.

DEFINITION 1. Let $f(z)=z+\sum_{n=2}^{\infty} a_{n} z^{n} \in A$ and satisfy $f(z) f^{\prime}(z) \neq 0$ in $0<|z|<1$. Set

$$
K(\lambda, \alpha, f(z))=\left(e^{i \lambda}-\alpha\right) z f^{\prime}(z) / f(z)+\alpha\left(z f^{\prime \prime}(z) / f^{\prime}(z)+1\right) .
$$

Then $f(z)$ is said to be $\alpha$ - $\lambda$-spiral-like of order $\beta$ if

$$
\operatorname{Re} K(\lambda, \alpha, f(z))>\beta \cos \lambda \quad(z \in U)
$$

where $\alpha \geqq 0,0 \leqq \beta<1,|\lambda|<\pi / 2$.

Remarks. (i) For $\alpha=0, S_{0}^{\lambda}(\beta)$ is the class of $\lambda$-spiral-like functions of order $\beta$.

(ii) For $\lambda=0=\beta$, we have $S_{\alpha}^{0}(0)$-the class of $\alpha$-star-like functions (of order zero); while $S_{\alpha}^{0}(\beta) \quad(\alpha \geqq 0,0 \leqq \beta<1)$ is the class of $\alpha$-starlike functions of order $\beta$.

In order to prove that $\alpha$ - $\lambda$-spiral-likeness of order $\beta(\alpha \geqq 0)$ implies $\lambda$-spiral-likeness of order $\beta$, we will need the following two lemmas: the first lemma is due to I. S. Jack [4] while the second is due to R. Libera [6].

Lemma A. Let $\omega(z)$ be regular in $U$ with $\omega(0)=0$. If there exists a $\zeta \in U$ such that $\operatorname{Max}_{|z| \leq|\zeta|}|\omega(z)|=|\omega(\zeta)|$, then $\zeta \omega^{\prime}(\zeta)=k \omega(\zeta)$ for some $k \geqq 1$.

Lemma B. The function $f(z) \in A$ is $\lambda$-spiral-like of order $\beta(0 \leqq \beta<1$, $|\lambda|<\pi / 2)$ if and only if there exists an $\omega(z)$ analytic satisfying $\omega(0)=0$, $|\omega(z)|<1$ such that

$$
e^{i \lambda} \frac{z f^{\prime}(z)}{f(z)}=\beta \cos \lambda+(1-\beta) \cos \lambda\left(\frac{1-\omega(z)}{1+\omega(z)}\right)+i \sin \lambda \quad(z \in U) .
$$


THEOREM 1. If $f(z) \in S_{\alpha}^{\lambda}(\beta) \quad(\alpha \geqq 0,0 \leqq \beta<1,|\lambda|<\pi / 2)$ then $f(z)$ is $\lambda$-spiral-like of order $\beta$.

Proof. Let

$$
e^{i \lambda} \frac{z f^{\prime}(z)}{f(z)}=\beta \cos \lambda+(1-\beta) \cos \lambda\left(\frac{1-\omega(z)}{1+\omega(z)}\right)+i \sin \lambda .
$$

Clearly, $\omega(0)=0$. In view of Lemma B, it suffices to show that $|\omega(z)|<1$. Simplifying (3), it follows that

$$
e^{i \lambda} \frac{z f^{\prime}(z)}{f(z)}=\frac{e^{i \lambda}\left\{1+\left(2 \beta e^{-i \lambda} \cos \lambda-e^{-2 i \lambda}\right) \omega(z)\right\}}{1+\omega(z)} .
$$

Differentiating (4) and using (1), we have

$$
\begin{aligned}
K(\lambda, \alpha, f(z))= & \beta \cos \lambda+(1-\beta) \cos \lambda\left(\frac{1-\omega(z)}{1+\omega(z)}\right)+i \sin \lambda \\
& +\alpha \frac{\left\{2 \beta e^{-i \lambda} \cos \lambda-e^{-2 i \lambda}\right\} z \omega^{\prime}(z)}{1+\left(2 \beta e^{-i \lambda} \cos \lambda-e^{-2 i \lambda}\right) \omega(z)}-\alpha \frac{z \omega^{\prime}(z)}{1+\omega(z)} .
\end{aligned}
$$

Suppose that there exists a $\zeta \in U$ such that $\operatorname{Max}_{|z| \leqq|\zeta|}|\omega(z)|=|\omega(\zeta)|=1$. Clearly $\omega(\zeta) \neq-1$. From Lemma $A$, there exists a $k \geqq 1$ such that $\zeta \omega^{\prime}(\zeta)=k \omega(\zeta)$. For this $\zeta$, we have

(6) $\operatorname{Re}(1-\omega(\zeta)) /(1+\omega(\zeta))=0, \quad \operatorname{Re} \zeta \omega(\zeta) /(1+\omega(\zeta))=k / 2$.

Also, for

$$
\begin{aligned}
m= & 2 \beta e^{-i \lambda} \cos \lambda-e^{-2 i \lambda} \\
\operatorname{Re} \frac{m \zeta \omega^{\prime}(\zeta)}{1+m \omega(\zeta)} & =\operatorname{Re} \frac{k\left(|m|^{2}+m \omega(\zeta)\right)}{1+|m|^{2}+2 \operatorname{Re} m \omega(\zeta)} \\
& =\operatorname{Re} \frac{k\left(|m|^{2}+\operatorname{Re} m \omega(\zeta)\right)}{1+|m|^{2}+2 \operatorname{Re} m \omega(\zeta)}
\end{aligned}
$$

Hence,

(8) $\operatorname{Re}\left(\frac{m \zeta \omega^{\prime}(\zeta)}{1+m \omega(\zeta)}\right)-\operatorname{Re}\left(\frac{\zeta \omega^{\prime}(\zeta)}{1+\omega(\zeta)}\right)=\frac{k\left(|m|^{2}-1\right)}{2\left(1+2 \operatorname{Re} m \omega(\zeta)+|m|^{2}\right)}$.

Thus, from (6), (7) and (8), it follows that

(9) $\operatorname{Re} K(\lambda, \alpha, f(z))=\beta \cos \lambda-\frac{2 k \beta(1-\beta) \alpha \cos ^{2} \lambda}{1+|m|^{2}+2 \operatorname{Re} m \omega(\zeta)}<\beta \cos \lambda$, 
contradicting the assumption that $f(z) \in S_{\alpha}^{\lambda}(\beta)$. Therefore $|\omega(z)|<1$ in $U$ and $f(z)$ is $\lambda$-spiral-like of order $\beta$.

COROLlaRY. If $f(z) \in S_{\alpha}^{\lambda}(\beta)$ then $f(z) \in S_{\gamma}^{\lambda}(\beta), 0 \leqq \gamma \leqq \alpha$.

Proof. By Theorem $1, f(z) \in S_{0}^{\lambda}(\beta)$. Suppose there exists a $\gamma, 0<\gamma<\alpha$, such that $f(z) \notin S_{\gamma}^{\lambda}(\beta)$. Then there is a $\zeta \in U$ for which

$$
\operatorname{Re}\left(\frac{\zeta f^{\prime \prime}(\zeta)}{f^{\prime}(\zeta)}+1-\frac{\zeta f^{\prime}(\zeta)}{f(\zeta)}\right) \leqq \frac{\beta \cos \lambda}{\gamma}-\frac{1}{\gamma} \operatorname{Re} \frac{\zeta f^{\prime}(\zeta)}{f(\zeta)}
$$

However, for $f(z) \in S_{\alpha}^{\lambda}(\beta)$,

(11) $0<-\beta \cos \lambda+\operatorname{Re} e^{i \lambda} \frac{\zeta f^{\prime}(\zeta)}{f(\zeta)}+\alpha \operatorname{Re}\left(\frac{\zeta f^{\prime \prime}(\zeta)}{f^{\prime}(\zeta)}+1-\frac{\zeta f^{\prime}(\zeta)}{f(\zeta)}\right)$.

Substituting (10) into (11), we obtain

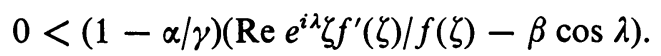

But $(1-\alpha / \gamma)<0$ implies $\operatorname{Re} e^{i \lambda} z f^{\prime}(z) \mid f(z)<\beta \cos \lambda$, contradicting the assumption that $f(z) \in S_{0}^{\lambda}(\beta)$. Thus, $f(z) \in S_{\gamma}^{\lambda}(\beta)$.

3. In this section, the author obtains an important integral representation for the elements of $S_{\alpha}^{\lambda}(\beta)$. Throughout this section $\alpha, \beta, \lambda$ will represent constants such that $\alpha>0,0 \leqq \beta<1,|\lambda|<\pi / 2$.

Definition 2. The function

$$
f(z)=\left[(\gamma+i \mu) \int_{0}^{z} \sigma(t)^{\gamma} t^{-1+i \mu} d t\right]^{1 /(\gamma+i \mu)}
$$

where $\sigma(t) \in S^{*}(0), \gamma>0, \mu$ real, $z \in U$ and the powers are meant as principal values, is called a Bazilevič function of type $\gamma+i \mu$. Denote the class of such functions by $B(\gamma+i \mu)$ [2].

Due to a result by Eenigenburg et al. [3], we know that each $f(z) \in B(\gamma+i \mu)$ is spiral-like. The functions that we will use in order to characterize the elements of $S_{\alpha}^{\lambda}(\beta)$ are those obtained when $\gamma=(\cos \lambda) / \alpha$ and $\mu=(\sin \lambda) / \alpha$.

Definition 3. A function $f(z) \in A$ is said to be a Bazilevič function of type $e^{i \lambda} / \alpha$ and order $\beta$ if

$$
f(z)=\left[\frac{e^{i \lambda}}{\alpha} \int_{0}^{z} \sigma(\zeta)^{(\cos \lambda) / \alpha \zeta-1+((i \sin \lambda) / \alpha)} d \zeta\right]^{\alpha e^{-i \lambda}}
$$

for some $\sigma(\zeta) \in S^{*}(\beta)$. Denote this by $f(z) \in B\left(e^{i \lambda} / \alpha, \beta\right)$.

Immediate from Definition 3 is

THEOREM 2. If $f(z) \in B\left(e^{i \lambda} / \alpha, \beta\right)$ then $f(z) \in S_{\alpha}^{\lambda}(\beta)$. 
Proof. For $f(z) \in B\left(e^{i \lambda} / \alpha, \beta\right)$, it follows from (12) that

$$
f^{\prime}(z)=\sigma(z)^{\cos \lambda / \alpha} z^{-1+((i \sin \lambda) / \alpha)} f(z)^{1-e^{i \lambda / \alpha}} .
$$

Taking the logarithmic derivative of (13) we obtain an expression for $\left[z f^{\prime \prime}(z) \mid f^{\prime}(z)\right]+1$. Substituting this into (1), we have

$$
K(\lambda, \alpha, f(z))=\cos \lambda z \sigma^{\prime}(z) / \sigma(z)+i \sin \lambda .
$$

Thus, $\operatorname{Re} K(\lambda, \alpha, f(z))>\beta \cos \lambda$ or $f(z) \in S_{\alpha}^{\lambda}(\beta)$.

Using the following lemma due to Basgöze and Keogh [1], a necessary and sufficient condition for $f(z)$ to be in $B\left(e^{i \lambda} / \alpha, \beta\right)$ is obtained.

Lemma C. A function $\sigma(\zeta) \in S^{*}(\beta)$ if and only if there exists a function $F(\zeta) \in S_{0}^{\lambda}(\beta)$ such that

$$
(\sigma(\zeta) / \zeta)^{\cos \lambda}=(F(\zeta) / \zeta)^{e^{i \lambda}} \quad(\zeta \in U) .
$$

Lemma 1. A function $f(z) \in B\left(e^{i \lambda} / \alpha, \beta\right)$ if and only if there exists a function $F(\zeta) \in S_{0}^{\lambda}(\beta)$ such that

$$
f(z)=\left[\frac{e^{i \lambda}}{\alpha} \int_{0}^{z}[F(\zeta)]^{i \lambda / \alpha \zeta-1} d \zeta\right]^{\alpha e^{-i \lambda}}
$$

where the powers are meant as principal values.

Proof. From Definition 3, $f(z) \in B\left(e^{i \lambda} / \alpha, \beta\right)$ if and only if there exists a $\sigma(\zeta) \in S^{*}(\beta)$ satisfying (12). However, a necessary and sufficient condition for $\sigma(\zeta) \in S^{*}(\beta)$ is that there exists an $F(\zeta) \in S_{0}^{\lambda}(\beta)$ satisfying (15). Thus, for $f(z) \in B\left(e^{i \lambda} / \alpha, \beta\right)$, we may obtain

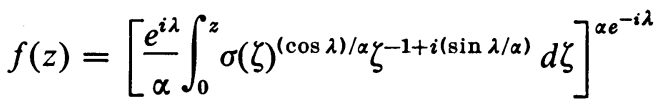

$$
\begin{aligned}
& =\left[\frac{e^{i \lambda}}{\alpha} \int_{0}^{z}\left(\frac{\sigma(\zeta)}{\zeta}\right)^{(\cos \lambda) / \alpha} \zeta^{-1+\left(e^{i \lambda / \alpha)}\right.} d \zeta\right]^{\alpha e^{-i \lambda}} \\
& =\left[\frac{e^{i \lambda}}{\alpha} \int_{0}^{z}[F(\zeta)]^{i \lambda / \alpha \zeta-1} d \zeta\right]^{\alpha e^{-i \lambda}},
\end{aligned}
$$

where $\sigma(\zeta)$ and $F(\zeta)$ are as above. Since each step in (17) is reversible, the result follows from this identity.

REMARK. From Lemma 1, a necessary and sufficient condition for $f(z) \in B\left(e^{i \lambda} / \alpha, \beta\right)$ is that

$$
F(z)=f(z)\left[z f^{\prime}(z) / f(z)\right]^{\alpha e^{-i \lambda}}
$$

where $F(z) \in S_{0}^{\lambda}(\beta)$. Also, $B\left(e^{i \lambda} / \alpha, \beta\right) \subset S_{\alpha}^{\lambda}(\beta)$. In order to obtain the 
characterization for functions $f(z) \in S_{\alpha}^{\lambda}(\beta)$, we consider the converse problem. Given $F(\zeta) \in S_{0}^{\lambda}(\beta)$ and $\alpha>0$, when is the solution to the differential equation (18) with boundary condition $f(0)=0$, a function that is $\alpha$ - $\lambda$-spiral-like of order $\beta$ ? Since (18) may be rewritten as $[F(z)]^{e^{-i \lambda / \alpha} / z=}$ $f^{\prime}(z) f(z)^{-1+\left(e^{i \lambda / \alpha)}\right.}$ we may perform the integration with boundary condition $f(0)=0$ to obtain

$$
f(z)=\left[\frac{e^{i \lambda}}{\alpha} \int_{0}^{z} \frac{[F(\zeta)]^{i \lambda / \alpha}}{\zeta} d \zeta\right]^{\alpha e^{-i \lambda}} .
$$

We will now show the proper definitions for which this formal solution is indeed an $\alpha$ - $\lambda$-spiral-like function of order $\beta$.

Lemma 2. Let $f(z) \in S_{\alpha}^{\lambda}(\beta)$. For $0<\gamma \leqq \alpha$, choose the branch of $\left[z f^{\prime}(z) \mid f(z)\right]^{\gamma^{-i \lambda}}$ equal to 1 when $z=0$. Then the function

$$
F_{\gamma}(z)=f(z)\left[z f^{\prime}(z) / f(z)\right]^{\gamma e^{-i \lambda}}
$$

is $\lambda$-spiral-like of order $\beta$.

Proof. We have

$$
e^{i \lambda} \frac{z F_{\gamma}^{\prime}(z)}{F_{\gamma}(z)}=e^{i \lambda} \frac{z f^{\prime}(z)}{f(z)}+\gamma\left(\frac{z f^{\prime \prime}(z)}{f^{\prime}(z)}+1-\frac{z f^{\prime}(z)}{f(z)}\right)=K(\lambda, \gamma, f(z)) .
$$

But by the corollary to Theorem 1 , we have that $f(z) \in S_{\alpha}^{\lambda}(\beta)$ implies $f(z) \in S_{\gamma}^{\lambda}(\beta)(0 \leqq \gamma \leqq \alpha)$. Therefore, $\operatorname{Re} e^{i \lambda} z F_{\gamma}^{\prime}(z) / F_{\gamma}(z)=\operatorname{Re} K(\lambda, \gamma, f(z))>$ $\beta \cos \lambda$ and $F_{\gamma}(z) \in S_{0}^{\lambda}(\beta)$.

Lemma 3. If $F(z)=z+A_{2} z+\cdots \in S_{0}^{\lambda}(\beta)$ then $F(z)$ may be expressed as

$$
F(z)=f(z)\left[z f^{\prime}(z) / f(z)\right]^{\alpha e^{-i \lambda}},
$$

where

$$
f(z)=\left[\frac{e^{i \lambda}}{\alpha} \int_{0}^{z}[F(\zeta)]^{i \lambda / \alpha \zeta-1} d \zeta\right]^{\alpha e^{-i \lambda}}
$$

is an $\alpha-\lambda$-spiral-like function of order $\beta$.

Proof. Let $h(z)=z^{-e^{i \lambda / \alpha}} \int_{0}^{z}[F(\zeta)]^{e^{i \lambda / \alpha} \zeta-1} d \zeta$. We have

$$
f(z)=z\left[\left(e^{i \lambda} / \alpha\right) h(z)\right]^{\alpha e^{-i \lambda}}
$$

so that if $h(z)$ is independent of the path of integration it will follow that $f(z)$ is well defined.

Since $F(z)=z\left(1+A_{2} z+\cdots\right) \in S_{0}^{\lambda}(\beta)$, we have that $\left(1+A_{2} z+\cdots\right)$ is 
nonzero in $U$. Thus, we may write

$$
\left(1+A_{2} z+\cdots\right)^{i \lambda / \alpha}=1+\sum_{n=1}^{\infty} c_{n} z^{n}
$$

for the power series expansion about $z=0$. From (22), it follows that

$$
\int_{0}^{z} F(\zeta)^{e^{i \lambda} / \alpha \zeta^{-1}} d \zeta=\alpha e^{-i \lambda} z^{i \lambda / \alpha}\left(1+\sum_{n=1}^{\infty} \frac{c_{n}}{\alpha e^{i \lambda} n+1} z^{n}+C\right) .
$$

To obtain a solution of (23) which is analytic and zero at the origin, take $C=0$. Thus, $h(z)=\alpha e^{-i \lambda}\left(1+\sum_{n=1}^{\infty} c_{n} z^{n} /\left(\alpha e^{i \lambda} n+1\right)\right)$ is independent of the path of integration so that $f(z)$ given by $(21)$ is well defined.

That $f(z)$ is $\alpha$ - $\lambda$-spiral-like of order $\beta$ is a consequence of Theorem 2 and Lemma 1. Thus, the lemma is proved.

By combining the results of Theorem 2, Lemma 2 and Lemma 3, we have

THEOREM 3. A necessary and sufficient condition for $f(z)$ to be in $S_{\alpha}^{\lambda}(\beta)$ is that $f(z)$ have the integral representation

$$
f(z)=\left[\frac{e^{i \lambda}}{\alpha} \int_{0}^{z}[F(\zeta)]^{i \lambda / \alpha \zeta^{-1}} d \zeta\right]^{\alpha e^{-i \lambda}}
$$

for some $F(\zeta) \in S_{0}^{\lambda}(\beta)$, where the powers are assumed to be principal values.

PROOF. If $f(z)$ is of the form (24), it follows immediately from Theorem 2 and Lemma 1 that $f(z) \in S_{\alpha}^{\lambda}(\beta)$. If $f(z) \in S_{\alpha}^{\lambda}(\beta)$, then-by Lemma 2 and Lemma $3-f(z)$ can be written in the form (24).

Note that we now have $B\left(e^{i \lambda} / \alpha, \beta\right)=S_{\alpha}^{\lambda}(\beta)$ for $\alpha>0,0 \leqq \beta<1,|\lambda|<\pi / 2$.

4. We conclude this paper with a determination of a distortion theorem and a rotation theorem for $f(z) / z$ whenever $f(z) \in M_{0}^{\lambda}(\beta)=M^{\lambda}(\beta)(0 \leqq$ $\beta<1,|\lambda|<\pi / 2)$.

For $f(z) \in M^{\lambda}(\beta)(0 \leqq \beta<1,|\lambda|<\pi / 2)$ there exists a $p(z) \in P$ such that

$$
e^{i \lambda} z f^{\prime}(z) / f(z)=(1-\beta) \cos \lambda p(z)+\beta \cos \lambda+i \sin \lambda .
$$

From (25) it follows that

$$
e^{i \lambda}\left(z f^{\prime}(z) / f(z)-1\right)=(1-\beta) \cos \lambda(p(z)-1) .
$$

Throughout this section $\lambda, \beta$ will denote constants satisfying $|\lambda|<\pi / 2$, $0 \leqq \beta<1$.

Using (26) we are able to obtain the convex hull of the image of $\log f(z) / z$ for fixed $z(|z|=r<1)$ when $f(z) \in M^{\lambda}(\beta)$.

THEOREM 4. If $f(z) \in M^{\lambda}(\beta)$ then the set of all possible values of $\log f(z) / z$ ( $z$ fixed, $|z|=r<1$ ) lies in the image of $|z| \leqq r$ under the map

$$
\omega(z)=\log \left[(1-\varepsilon z)^{-2(1-\beta) e^{-i \lambda} \cos \lambda}\right], \quad|\varepsilon|=1 .
$$


Proof. Dividing both sides of (26) by $z \neq 0$, integrating from 0 to $z$ and simplifying, we have

$$
\log \frac{f(z)}{z}=(1-\beta) e^{-i \lambda} \cos \lambda \int_{0}^{z} \frac{p(\zeta)-1}{\zeta} d \zeta .
$$

Since $p(z) \in P$, Herglotz's theorem [12] may be applied to obtain

$$
p(\zeta)=\int_{-\pi}^{\pi} \frac{1+\zeta e^{i t}}{1-\zeta e^{i t}} d \mu(t)
$$

where $\mu(t)$ is nondecreasing in $[-\pi, \pi]$ and $\int_{-\pi}^{\pi} d \mu(t)=1$. From (28), it follows that

$$
\frac{p(\zeta)-1}{\zeta}=\int_{-\pi}^{\pi} \frac{2 e^{i t}}{1-\zeta e^{i t}} d \mu(t) .
$$

Substituting (29) into (27), we obtain

$$
\log \frac{f(z)}{z}=-2(1-\beta) e^{-i \lambda} \cos \lambda \int_{-\pi}^{\pi} \log \left(1-e^{i t} z\right) d \mu(t) .
$$

Let $q(z, t)=\log \left(1-e^{i t} z\right)^{-2(1-\beta) e^{-i \lambda} \cos \lambda}$. Then $\operatorname{Re}\left\{1+z q^{\prime \prime}(z, t) / q^{\prime}(z, t)\right\}=$ $\operatorname{Re}\left[1 /\left(1-z e^{i t}\right)\right]>\frac{1}{2}$. Thus, $q(z, t)$ is univalent in $z$ and maps $|z| \leqq r<1$ onto a convex domain $E$, independent of $t$. From (30), we know that for fixed $z(|z|=r<1)$ the points of $\log f(z) / z$ lie in the convex hull of $E$, denoted con $E$. However, since $E$ is convex, $E=\operatorname{con} E$ and the points of $\log f(z) / z(z$ fixed, $|z|=r<1)$ lie in the convex image of $|z| \leqq r$ under the mapping $\omega(z)$ given by (27).

REMARKS. (i) For

$$
\log f_{t}(z) / z=\log \left[\left(1-e^{i t} z\right)^{-2(1-\beta) e^{-i \lambda} \cos \lambda}\right] \quad(-\pi \leqq t<\pi),
$$

we have

$$
f_{t}(z)=z\left(1-e^{i t} z\right)^{-2(1-\beta) e^{-i \lambda} \cos \lambda} .
$$

These $f_{t}(z)$-for different $t$-are the extremal functions for Theorem 4 .

(ii) We have

$$
\log \left|f_{t}(z) / z\right|=\operatorname{Re} \log \left[\left(1-e^{i t} z\right)^{-2(1-\beta) e^{-i \lambda} \cos \lambda}\right]
$$

and

$$
\arg f_{t}(z) / z=\operatorname{Im} \log \left[\left(1-e^{i t} z\right)^{-2(1-\beta) e^{-i \lambda} \cos \lambda}\right] .
$$

Also, for $z=r e^{i \theta}(0<r<1,0 \leqq \theta<2 \pi)$ and $\eta=\theta+t$, we have

$$
\log \left[\left(1-e^{i t} z\right)^{-2(1-\beta) e^{-i \lambda} \cos \lambda}\right]=T(r, \eta, \lambda, \beta)+i S(r, \eta, \lambda, \beta)
$$


where

$T(r, \eta, \lambda, \beta)$
(34) $=(1-\beta) \cos \lambda\left\{2 \sin \lambda \arctan \frac{r \sin \eta}{1-r \cos \eta}-\cos \lambda \log \left(1-2 r \cos \eta+r^{2}\right)\right\}$

and

$S(r, \eta, \lambda, \beta)$
${ }^{(35)}=(1-\beta) \cos \lambda\left\{2 \cos \lambda \arctan \frac{r \sin \eta}{1-r \cos \eta}+\sin \lambda \log \left(1-2 r \cos \eta+r^{2}\right)\right\}$.

Since $\left\{f_{t}(z) \mid t \in[-\pi, \pi]\right\}$ represent the extremal functions of Theorem 4 , the distortion and rotation theorems follow from (31) through (35).

THEOREM 5. If $f(z) \in M^{\lambda}(\beta)$, for fixed $z(|z|=r<1), T\left(r, \eta_{1}, \lambda, \beta\right) \leqq$ $\log |f(z) / z| \leqq T\left(r, \eta_{2}, \lambda, \beta\right)$ where

$$
\eta_{1,2}=2 \tan ^{-1}\left\{\frac{-\cot \lambda \mp\left(\operatorname{cosec}^{2} \lambda-r^{2}\right)^{1 / 2}}{1+r}\right\} .
$$

Proof. It suffices to determine the bounds for $\log \left|f_{t}(z) / z\right|$ where $f_{t}(z)$ are the extremal functions for Theorem 4. Since $\log \left|f_{t}(z) / z\right|=T(r, \eta, \lambda, \beta)$ is a real-valued function of $\eta$, we may determine the maximum and minimum points by using elementary calculus. It follows that $\partial T / \partial \theta=0$ for $\eta_{1,2}$ given in (26). By examining $\partial^{2} T / \partial \theta^{2}$, we find that $\partial^{2} T / \partial \theta^{2}$ is positive for $\eta_{1}$ and negative for $\eta_{2}$. The result follows.

THEOREM 6. If $f(z) \in M^{\lambda}(\beta)$ ( $z$ fixed, $\left.|z|=r<1\right)$, then

where

$$
S\left(r, \eta_{3}, \lambda, \beta\right) \leqq \arg f(z) / z \leqq S\left(r, \eta_{4}, \lambda, \beta\right)
$$

$$
\eta_{3,4}=2 \tan ^{-1}\left\{\frac{\tan \lambda \mp\left(\sec ^{2} \lambda-r^{2}\right)^{1 / 2}}{1+r}\right\} .
$$

Proof. This follows immediately by applying the same procedures as in the proof of Theorem 5 to $\arg f_{t}(z) / z=S(r, \eta, \lambda, \beta)$. Here $S(r, \eta, \lambda, \beta)$ is a real-valued function of $\eta$ whose derivative is zero for $\eta_{3,4}$-given by (37). The second derivative of $S$ is positive for $\eta_{3}$ and negative for $\eta_{4}$ from which the result follows.

REMARK. For $\beta=0$, Theorems 5 and 6 give us the known results for $\hat{\lambda}$-spiral-like functions of order $\beta[13]$.

\section{REFERENCES}

1. T. Basgöze and F. R. Keogh, The Hardy class of a spiral-like function and its derivative, Proc. Amer. Math. Soc. 26 (1970), 266-269. MR 41 \#8680. 
2. I. E. Bazilevič, On a case of integrability in quadratures of the Loewner-Kufarev equation, Mat. Sb. 37 (79) (1955), 471-476. (Russian) MR 17, 356.

3. P. Eenigenburg, S. Miller, P. Mocanu and M. Reade, On a class of Bazilevič functions, Notices Amer. Math. Soc. 19 (1972), A706. Abstract \#72T-B293.

4. I. S. Jack, Functions starlike and convex of order $\alpha$, J. London Math. Soc. (2) 3 (1971), 469-474. MR 43 \#7611.

5. R. Libera, Univalent $\alpha$-spiral functions, Canad. J. Math. 19 (1967), 449-456. MR 35 \#5599.

6. S. S. Miller, Distortion properties of alpha-starlike functions, Proc. Amer. Math. Soc. 38 (1973), 311-318.

7. S. S. Miller, P. T. Mocanu and M. O. Reade, All $\alpha$-convex functions are univalent and starlike, Proc. Amer. Math. Soc. 37 (1973), 553-554.

8. - Bazilevič functions and generalized convexity, Rev. Roumaine Math. Pures Appl. (to appear).

9. P. T. Mocanu, Une propriété de convexité généralisée dans la théorie de la représentation conforme, Mathematica (Cluj) 11 (34), (1969), 127-133. MR 42 \#7881.

10. P. T. Mocanu and M. O. Reade, On generalized convexity of conformal mappings, Rev. Roumaine Math. Pures Appl. 16 (1971), 1541-1544.

11. R. Nevanlinna, Eindeutige analytische Funktionen, Die Grundlehren der math. Wissenschaften, Band 46, Springer-Verlag, Berlin, 1936; English transl., Die Grundlehren der math. Wissenschaften, Band 162, Springer-Verlag, Berlin and New York, 1970. MR 43 \#5003.

12. E. Silvia, Classes related to $\alpha$-starlike functions, Ph.D. Dissertation, Clark University, Worcester, Mass., 1972.

13. R. Singh, A note on spiral-like functions, J. Indian Math. Soc. 33 (1969), 49-55. MR 41 \#454.

14. L. Spaček, Prispevek $k$ teorii funkei prostych, Casopis Pěst. Mat. 62 (1933), 12-19.

Department of Mathematics, Clark University, Worcester, Massachusetts 01610

Current address: Department of Mathematics, University of California, Davis, California 95616 